\title{
Influence of particle charging on TEOM measurements in the presence of an electrostatic precipitator
}

\author{
N. K. Meyer ${ }^{1, *}$, A. Lauber ${ }^{2}$, T. Nussbaumer ${ }^{2}$, and H. Burtscher ${ }^{1}$ \\ ${ }^{1}$ Institute for Aerosol and Sensor Technology, University of Applied Sciences, Windisch, Switzerland \\ ${ }^{2}$ Bioenergy Research Group, University of Applied Sciences, Lucerne, Horw, Switzerland \\ *now at: Laboratory for Energy Systems Analysis, Paul Scherrer Institut, Switzerland
}

Received: 12 September 2008 - Published in Atmos. Meas. Tech. Discuss.: 8 December 2008

Revised: 26 February 2009 - Accepted: 12 March 2009 - Published: 30 March 2009

\begin{abstract}
The efficiency of an electrostatic precipitator (ESP) for reducing wood combustion emissions was investigated. Real-time measurements were conducted by directly reading the change in frequency of the tapered element in a Thermo Scientific 1400a TEOM. These measurements have been shown to be influenced by the charge on the aerosols reaching the tapered element such that the TEOM overestimates mass concentration. This electrostatic effect was crosschecked with particle mass concentration and particle number concentration measurements where no influence was observed. Placing a radioactive neutraliser prior to the TEOM leads to agreement between observed ESP efficiencies as measured by both the TEOM, mass filters and a CPC.
\end{abstract}

\section{Introduction}

Particulate emissions from domestic wood burning appliances can be reduced by placing an electrostatic precipitator (ESP) directly in the emission stack. An ESP generates corona ions that charge an aerosol, leading to forced electrophoretic deposition of the particles. The efficiency of an ESP is heavily dependant upon ion concentration, field strength between the active and grounding electrodes, temperature and stack velocity (residence time). Due to the transport mechanisms in the ESP, the precipitation is mainly influenced by the particle size, and also by particle morphology and composition. Measurement of efficiency is most readily determined by comparing samples collected while the filter is off and then on. A common method for this is the comparison of mass concentrations collected using heated gravimetric filters. Various international emission monitoring standards dictate that sampling of both ambient and com-

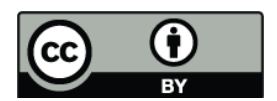

Correspondence to: N. K. Meyer (nicmeyer@yahoo.co.uk) bustion generated aerosols must be conducted via gravimetric analysis. This technique is both labour intensive and time consuming and subject to experimental error. Alternative methods for determination of mass concentration include the Tapered Element Oscillating Microbalance (TEOM), beta absorption and optical detection (Burtscher, 2005). In contrast to the gravimetric determination of particle mass collected on filters these methods offer approximations to realtime data and (excluding the TEOM) do not directly measure mass. Beta attenuation relies upon attenuation of the signal from a known beta source by material deposited between the source and a suitable detector (Baron and Willeke, 2001). The signal attenuation is known to be species dependant, which can lead to variances in observed results. Water vapour is prevented from positively biasing mass measurements by passing the aerosol through heated sample lines, however this technique is known to negatively bias mass measurements via significant volatilisation of (semi-) volatile species from which the aerosol is comprised; as such the validity of the method is limited to controlled situations. Alternately, optical methods rely upon scattering, extinction and absorption to determine the volume of an aerosol (Baron and Willeke, 2001). The sensitivity of particle detection when using optical instruments rapidly decreases for small particles. Assuming constant particle density and correcting for shape factors incurred by the prevalence of non-spherical agglomerates leads to at best, an estimation of the mass concentration of an aerosol. When measuring ambient aerosols, optical methods (e.g.; Dust Trak, TSI Inc.) can be portable, simple to use and provides good time resolution data however, based upon particle morphology, their use should be carefully considered when measuring combustion emissions. A more suitable instrument for this task would be the TEOM. The TEOM collects particles on a filter that is seated on an oscillating tapered element in a heated environment $\left(50^{\circ} \mathrm{C}\right)$. The purpose of the temperature-controlled environment is to create reproducible measurement conditions. An increase in

Published by Copernicus Publications on behalf of the European Geosciences Union. 
deposited mass is observed as a reduction in the square of the frequency of oscillation of the system. Given its simple operating principle and reproducibility, the TEOM is accepted as an NIST standard for mass measurement. Measurements conducted with the TEOM are neither influenced by shape factors nor particle densities as the deposited mass is directly related to the measurement signal. It is known however that TEOM measurements are influenced by the composition of the aerosol. Volatile species (organics and $\mathrm{NH}_{4} \mathrm{NO}_{3}$ ) are removed due to the heated filter and sampling environment within the TEOM. A US EPA correction of 1.03 at standard temperature and pressure has been developed to correct for these volatile losses however its use is limited to ambient monitoring (EPA, 1990). Investigations of this correction factor in practise has been shown that it underestimates the influence of volatiles while experiences within a UK monitoring network suggest that correction factors of approximately 1.3 should be used at ambient conditions. These correction factors are are known to vary with season and locations as source contributions vary between rural and urban regions (Green et al., 2001; Green and Fuller, 2006). Our investigations have shown that the TEOM is also influenced by the charge loading on the measured sample. When utilising electrostatic methods for measuring aerosols, for example with a Scanning Mobility Particle Sizer (SMPS), an aerosol neutralizer is used to bring about charge equilibrium. As the TEOM does not rely upon electrostatic characterisation of particle, use of a neutraliser is not prescribed. However, field analysis of the TEOM, gravimetric filter and a condensation particle counter (CPC) has indicated that the absence of a neutraliser in the sample train lead to an artificial overestimation of particle mass concentration when the ESP is on.

\section{Experimental}

Combustion emissions were generated using an automatic wood pellet boiler. The pellet boiler was chosen as the emission source because its operating parameters were well understood and its emissions well characterised. When operated under steady state combustion conditions its emissions were constant. It is known that the proportion of salts found in emissions from automatic wood boilers operated under ideal conditions is high whereas the organic fraction has been reported to be low when compared with emissions from a conventional log wood stove. Under ideal combustion conditions (Nussbaumer, 2003) particulate emissions consist of mainly inorganic matter $(\mathrm{KCl}, \mathrm{CaOH}$ etc.) with organic carbon (OC) contributing between 1 and $5 \%$ to total mass, while particles from incomplete combustion consist mainly of soot and organic carbon with small amounts of inorganic matter (Nussbaumer and Hasler, 1999; Oser et al., 2000; Klippel and Nussbaumer, 2006). This facet of the combustion emissions enabled observation of the electrostatic effect upon mass concentrations without the influence of losses attributed

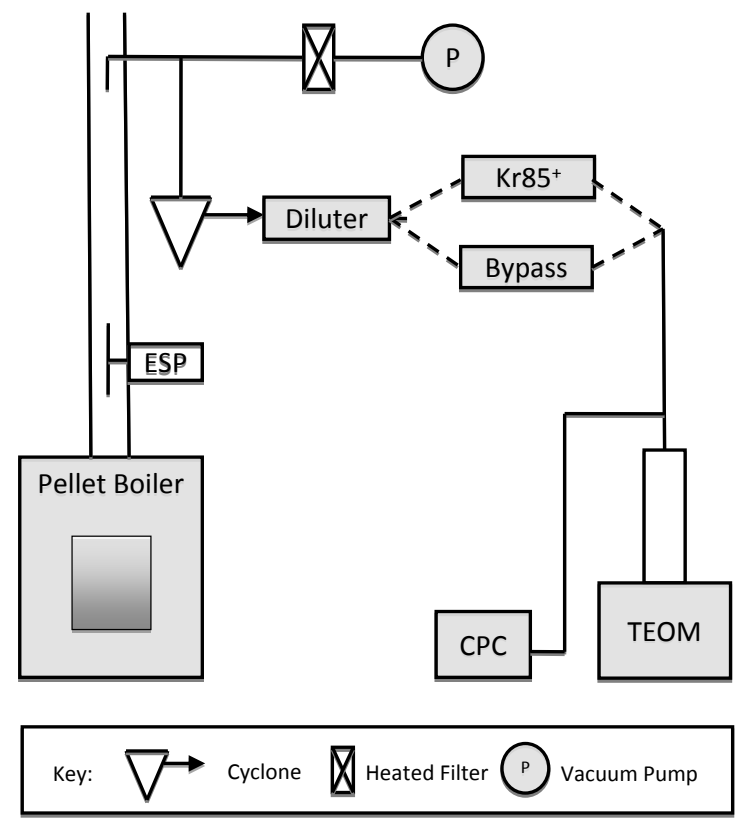

Fig. 1. Experimental setup.

to the volatilisation of condensable materials typically observed when using log wood stoves. Monitoring of combustion gases and conditions was maintained throughout the experiments to ensure that steady state combustion conditions were maintained (i.e., excess air $\lambda=1.55$ ). A simplified experimental setup is depicted in Fig. 1. Emissions generated by the boiler pass into the emissions stack at a constant velocity $(1 \mathrm{~m} / \mathrm{s})$. The ESP (built in-house) was situated a distance of two metres above the boiler and operated over a range of $12-14 \mathrm{kV}$. Filter samples were collected from the emission stack a distance of $0.5 \mathrm{~m}$ from the ESP and according to the VDI guideline concerning measurement of particulate mass, VDI2066. Filters were preconditioned at $180^{\circ} \mathrm{C}$ for $1 \mathrm{~h}$ and then stored in a silica gel desiccator for a period of $8 \mathrm{~h}$. During sampling, filters were held at a temperature of $160^{\circ} \mathrm{C}$ to prevent condensation of volatile species. The sample was collected through an isokinetic probe having diameter of $14 \mathrm{~mm}$ and sample flow of $1 \mathrm{~m} / \mathrm{s}$; all results (filter and TEOM) were normalised to $13 \% \mathrm{O}_{2}$. Normalisation to a specified oxygen concentration permits comparison of results between experiments conducted under different conditions. Raw concentrations observed in the emissions stack would saturate the TEOM and as such a dual stage heated ejector dilution unit is used to reduce observed concentrations (average dilution $\sim 150$ ). The first stage was heated to $150^{\circ} \mathrm{C}$ and supplied with heated, dry, hydrocarbon and particle free compressed air. The second stage dilution is supplied with the same air, albeit cold. $\mathrm{A} \mathrm{PM}_{10}$ cyclone is used to separate out any larger fractions present. After dilution the sample is then passed to the TEOM and CPC (TSI-3025) either through the Kr85+ neutraliser (TSI, High flow, 3012) or an equivalent length of $10 \mathrm{~mm}$ stainless steel tube. 


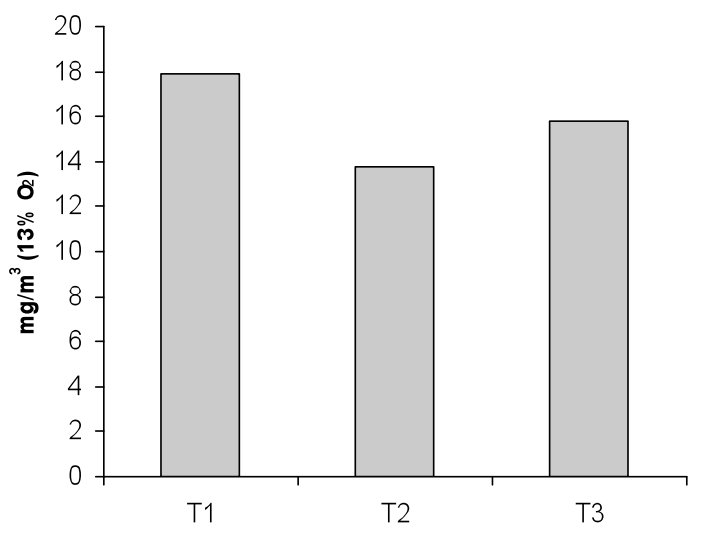

Fig. 2. Mass concentrations from a pellet boiler as measured by the TEOM with the ESP off. Each sample was collected over a period of $\sim 25 \mathrm{~min}$ at a dilution ration of $\sim 150$. The average mass concentration was $\sim 16 \mathrm{mg} / \mathrm{m}^{3}$.

Table 1. Summary of test conditions used when measuring with the TEOM.

\begin{tabular}{lcc}
\hline & ESP & $\mathrm{Kr}^{+}$Source \\
\hline 1. Off/no rad & off & bypass \\
2. Off/rad & off & inline \\
3. On/no rad & on & bypass \\
4. On/rad & on & inline \\
\hline
\end{tabular}

\section{Results}

\subsection{TEOM measurement characteristics}

To determine repeatability between the TEOM measurements three burn cycles were measured under ideal combustion conditions $(\lambda=1.55)$ with the ESP off (Fig. 2). The results were normalised to $13 \% \mathrm{O}_{2}$ (Normalisation factor $\sim 0.59$ ) and in-stack concentrations were calculated using Eq. (1) where $D$ is the dilution ratio $(\sim 150)$. The average sample time for each measurement was $25 \mathrm{~min}$.

$$
\left(\frac{\mathrm{mg}}{\mathrm{m}^{3}}\right)_{\text {In-Stack (normalised) }}=D \times\left(\frac{\mathrm{mg}}{\mathrm{m}^{3}}\right)_{\mathrm{TEOM} \text { (normalised) }}
$$

\subsection{Influence of the ESP on TEOM measurements}

To test the influence of the ESP on the TEOM measurements four different conditions were decided upon (summarised in Table 1). Average results and standard deviations from 6 tests under each condition are presented (Fig. 3). Maximum emissions (when the ESP was off) are shown to be $\sim 16 \mathrm{mg} / \mathrm{m}^{3}$. When the ESP was operating the particle concentration was measured to be $\sim 12.5 \mathrm{mg} / \mathrm{m}^{3}$. Placing the neutraliser inline was observed to influence the measurements such that the measured mass concentration reduced to $\sim 3 \mathrm{mg} / \mathrm{m}^{3}$. Given

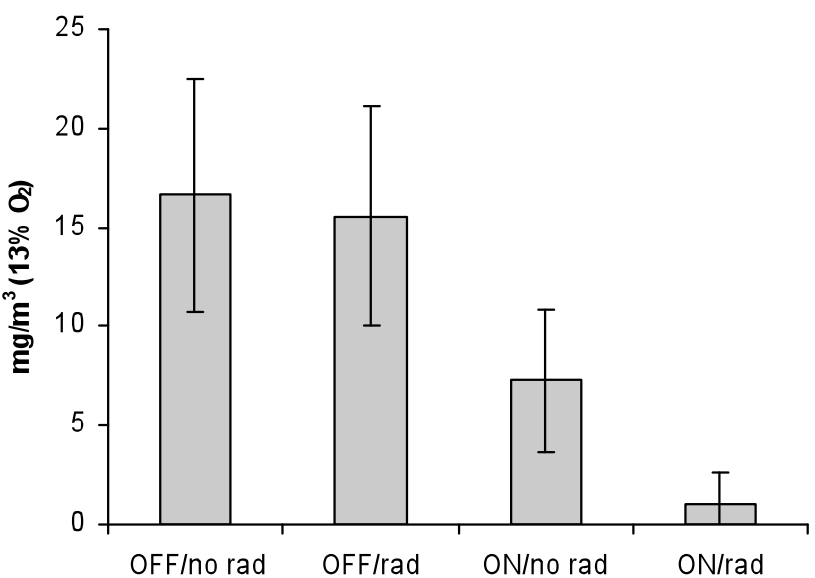

Fig. 3. Summary data - average TEOM results collected during measurements with the pellet boiler.

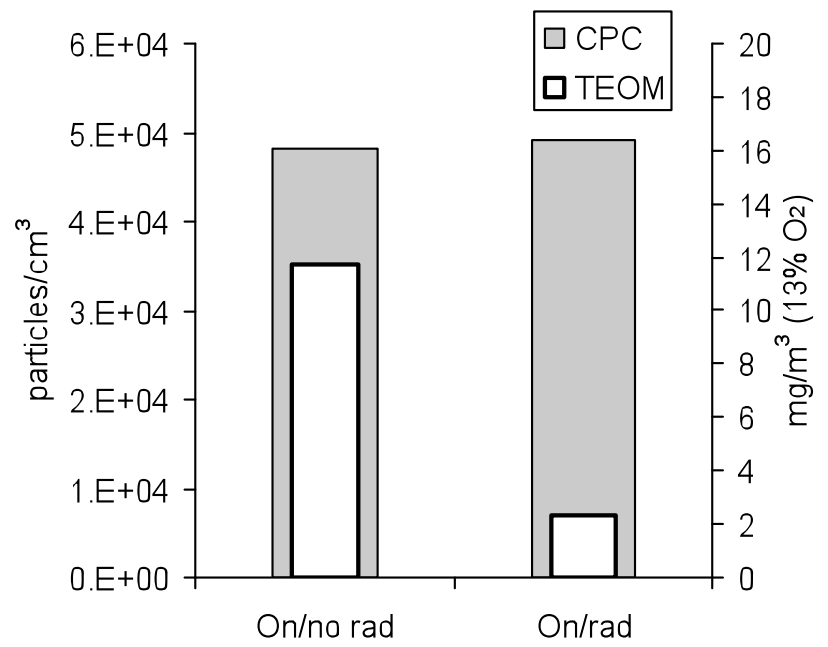

Fig. 4. Comparison of CPC and TEOM data when the Kr85+ source is either bypassed $(\mathrm{On} / \mathrm{no} \mathrm{rad})$ or inline $(\mathrm{On} / \mathrm{rad})$.

that the measurement characteristics of the TEOM are solely linked to mass accumulated on its collection substrate, this effect is both interesting and significant.

To determine whether the increase in mass observed in the absence of a neutraliser could be correlated with an increase in particle number concentration a CPC was positioned at the same measurement point as the TEOM. Results for this test can be seen in Fig. 4. The CPC number concentrations for both cases are shown in gray and the TEOM results in white. Number concentration as measured with the CPC remains constant whether the neutraliser is present or absent in the sample path. Mass concentration however, was observed to decrease when the neutraliser was placed inline, yet remain high in its absence. As a reference, total number concentrations measured when the ESP was off were an order of magnitude higher than those presented in Fig. 4. As such 


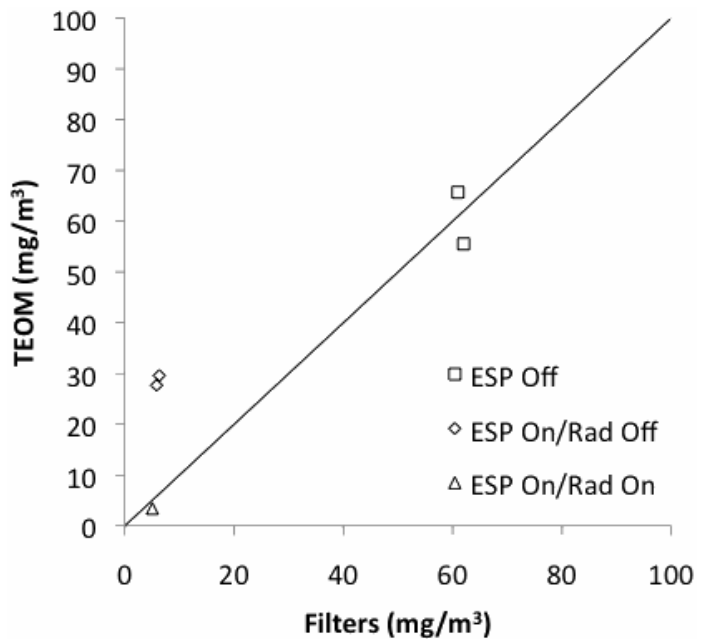

Fig. 5. Comparison of filter and TEOM measurements. Under normal condtions $\sim 1: 1$ agreement exists. Activating the ESP lead to significant oversetimation of mass concentration by the TEOM. This effect is counteracted by placing a high-flow neutraliser inline prior to the TEOM.

the particulate removal efficiency of the ESP as measured by both the TEOM and CPC was found to be $\sim 90 \%$. The results in Fig. 4 suggest that the charge induced on an aerosol does lead to a substantial overestimation of mass measured by the TEOM. Finally, measurements were conducted directly comparing the relationship between particle mass concentration measured using either filters or the TEOM (Fig. 5). When running the pellet boiler under normal operating conditions close agreement was observed between filter measurements and TEOM measurements of particulate mass concentration. However, when the ESP was activated, TEOM measurements were shown to greatly overestimate concentration as given by the filters. Placing the neutraliser inline prior to the TEOM removed the observed effect. This result further supports observations that TEOM measurements are indeed influenced by high particle charge loadings.

\section{Discussion}

Mass concentrations reported by the TEOM are derived from the oscillating frequency of its tapered element. Further confirmation that the observed measurement artefact is an electrostatic effect can be gained via analysis of the change in operating frequency of the instrument. Duplicate results collected during a single sampling day are presented in Fig. 6 . The measurement conditions presented refer to cases where the ESP is off, the ESP is on (without neutraliser) and the ESP is off (with neutraliser present). The differential of the frequency of the tapered element (df) reduces in this order according to the deposited mass. The case where the ESP is off shows the most negative gradient, this corresponds to the

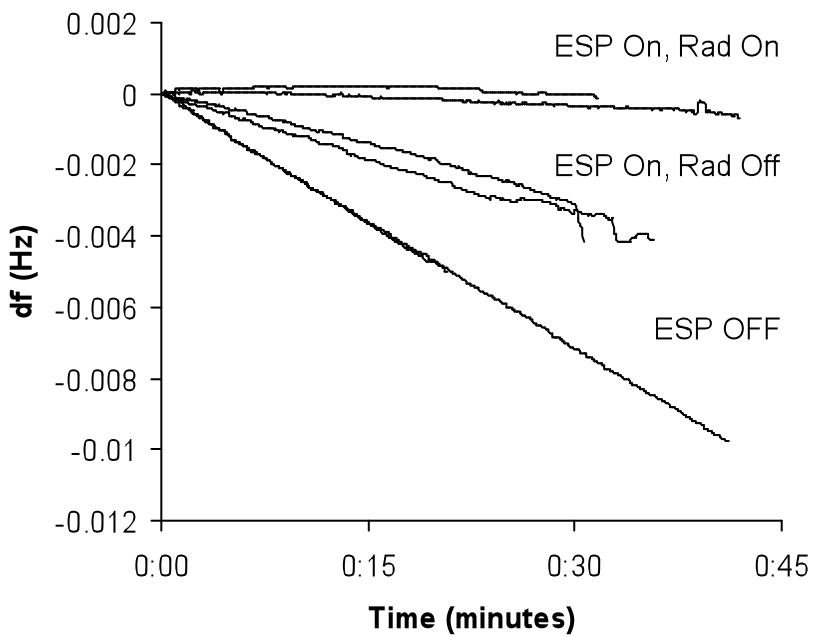

Fig. 6. Variation in tapered element frequency (df) during burn cycles. Larger reductions in frequency indicate larger deposited masses. Frequency reductions for three different conditions are shown.

largest mass deposition, while the case where the ESP is on and the neutraliser present shows a gradient closest to zero, corresponding to the lowest mass deposited. Between these two lines lays the response of TEOM frequency to the particles charged by the ESP. The gradient of this line is $\sim-10$ times that observed for the neutralised particles and approximately half that of the maximum observed mass. This analysis indicates a force (which can be attributed to particle charge) is acting on the oscillator. The TEOM manual indicates that the tapered element is seated between opposing field electrodes while Baron and Willeke (2001) state that the element is coated in a conductive material so that oscillation can be induced in the glass element. It is unclear whether only a portion or the whole of the element is conductive. If the former is the case, it is possible that collected charge could contribute to the observed reduction in oscillator frequency.

\section{Conclusions}

The influence of particle charging on TEOM measurements was investigated while conducting ESP efficiency tests on emissions from a domestic pellet boiler. Activating the ESP led to an increase in observed mass measured by the TEOM. This was inconsistent with values obtained during parallel TSP tests conducted using mass filter testing. This overestimation of mass by the TEOM was negated by placing a high flow neutraliser inline prior to the TEOM. Under optimal combustion conditions, ESP efficiency was found to be $94 \%$ when using the radioactive source and $55 \%$ when not. This variance is thought to be an artefact induced by the charge loading on the particles and could be negated by placing an 
aerosol neutraliser inline prior to the TEOM. This electrostatic effect was confirmed to be an artefact by comparing results against standard methods of measuring particulate number and mass concentrations (filters and CPC respectively) Further analysis of the frequency read directly from the tapered element of the instrument indicates that some dampening of the oscillator is observed. Given the high number of in-use TEOM's in long-term monitoring programs worldwide, this effect demands further investigation.

Edited by: A. Wiedensohler

\section{References}

Baron, P. A. and Willeke, K. (Eds.): Aerosol Measurement: Principles, Techniques and Applications, Wiley Interscience, 2001.

Burtscher, H.: Physical characterization of particulate emissions from diesel engines: a review, J. Aerosol Sci., 36(7), 896-932, 2005.

EPA: Ambient Air Monitoring Reference and Equivalent Methods Designation, US Environmental Protection Agency, Federal Register, 55, 43406-43407, 1990.

Chen, J. and Davidson, J. H.: Model of the Negative DC Corona Plasma: Comparision to the Positive DC Corona Plasma, Plasma Chem. Plasma Process, 23(1), 83-102, 2002.
Green, D., Fuller, G., and Barratt, B.: Evaluation of the TEOM "correction factors" for assessing EU Stage 1 limit values for PM10, Atmos. Environ., 35, 2589-2593, 2001.

Green, D. and Fuller, G. W.: The implications of tapered element oscillating microbalance (TEOM) software configuration on particulate matter measurements in the UK and Europe, Atmos. Environ., 40, 5608-5616, 2006.

Klippel, N. and Nussbaumer, T.: Feinstaubbildung in Holzfeuerungen und Gesundheitsrelevanz von Holzstaub im Vergleich zu Dieselruss, 9th Wood Energy Symposium, ETH, Zurich, Switzerland, 2006.

Nussbaumer, T.: Combustion and co-combustion of biomass: Fundamentals, technologies and primary measures for emission reduction, Energ. Fuel., 17, 1510-1521, 2003.

Nussbaumer, T. and Hasler, P.: Bildung und Eigenschaften von Aerosolen aus Holzfeuerungen, Holz als Roh- und Werkstoff, 57, 13-22, 1999.

Oser, M., Nussbaumer, T., Schweizer, B., Mohr, M., and Figi, R.: Untersuchung der Einflüsse auf die Partikelemissionen in einer Unterschubfeuerung, 6th Wood Energy Symposium, ETH, Zurich, 2000. 